\title{
A clinical approach of neuropathic pain in piriformis syndrome: case presentation and rehabilitation particularities
}

\section{VĂCĂRAȘ Vitalie ${ }^{1,2}$, FRUNZE Sorina ${ }^{2}$, CORDOȘ Mihai Adrian², PERCIULEAC Tatiana ${ }^{2}$ MURESANU Fior Dafin ${ }^{1,2}$}

Corresponding author: FRUNZE Sorina, E-mail: sorina frunze@yahoo.com

1. "Iuliu Haţieganu” University of Medicine and Pharmacy Cluj-Napoca, Romania 2. Cluj-Napoca County Emergency Hospital, Neurology Department, Cluj-Napoca, Romania

\begin{abstract}
Piriformis syndrome is a rare cause of sciatica and buttock pain and is usually considered as a diagnosis of exclusion for patients with a history of trauma. It usually presents without abnormalities regarding sensory and motor functions and tends to have a favorable evolution with pharmacological treatment. We present the case of a patient with posttraumatic piriformis syndrome who came to our department with significant pain and muscle weakness. The diagnosis was confirmed using clinical tests, imaging techniques and electroneurography. The patient did not respond to pharmacologic treatment alone and therefore surgery was performed. Surgery along with pharmacological treatment, complementary pain treatment and physical rehabilitation provided a favorable outcome.
\end{abstract}

Keywords: neurology, rehabilitation, piriformis syndrome, deep gluteal syndrome, traumatic neuropathy, neuropathic pain,

\section{Introduction}

The syntagm "Piriformis syndrome" can sometimes prove ambiguous, as it has multiple descriptions in current literature (1). Chronic pain localized in the lower limb, involving the areas innervated by the sciatic nerve, which is sometimes associated with lower back pain, is commonly called "sciatica" (2). For a long period of time it was wrongly thought that sciatica is only caused by the compression of the sciatic nerve by a protruding intervertebral disc. Nowadays this term is used to characterize compression of the sciatic nerve with various etiologies followed by the characteristic pain (2). Piriformis syndrome is responsible for $0.3-6 \%$ of sciatica cases (1).

In the year 1928, Yoemans was the first to propose and publish a correlation between sciatica and the piriformis muscle. The expression "piriformis syndrome", still used today, was later introduced in 1947 by Robinson et al (3). Only recently a new entity was described in literature, the "deep gluteal syndrome", which is currently preferred to describe pain that originates in the gluteal region and radiates into the territory of innervation of the sciatic nerve (4).

The sciatic nerve is formed in the pelvis from the L4 to S3 segments of the sacral plexus. It is the largest nerve in humans, having a diameter of $2 \mathrm{~cm}$ at the greater sciatic foramen, where it leaves the pelvis to enter the gluteal region. It passes underneath the piriformis muscle and over the superior gemellus, inferior gemellus and internal obturator muscles. The piriformis muscle has its origin on the anterior part of the sacrum, it passes laterally through the greater sciatic foramen and is inserted into the upper border of the greater trochanter. The muscle is an external rotator of the thigh when the thigh is extended on the abdomen, and an abductor of the thigh when the thigh is flexed on the abdomen (5). A dissection study of 240 cadavers performed by Beaton and Anson (6) evidenced six possible anatomical variations involving the piriformis muscle in relation to the sciatic nerve. The most frequent case was that of an undivided sciatic nerve that passes underneath the piriformis muscle, while in the other cases the fibers of the sciatic nerve were divided and passed through and underneath the muscle or over and underneath the muscle. The nerve can also pass as a whole through the piriformis muscle. Anatomical variations in which the nerve passes as a whole over the piriformis muscle, or is divided to pass over and through the piriformis muscle were not observed in the study, but were hypothesized as being possible. Variations regarding the tendon of the piriformis muscle were also observed (5), involving its diameter (ranging from 3 to $9 \mathrm{~mm}$ ) and its insertion. In about half of the cases observed, the tendon was inserted, as we mentioned above, on the upper border of the greater trochanter, but in about one third of the cases it was shown to fuse with the tendons of the superior gemellus and internal obturator muscles proximally to the insertion point. Other possibilities included its fusion with the tendons of the internal obturator and gluteus medius muscles in one fifth of the cases and its fusion with the gluteus medius muscle in a minority of cases (7). All these anatomical variations can have important clinical consequences, involving the mechanism of compression and the therapeutic approach. The causes that lead to the aforementioned piriformis syndrome are some of the multiple anatomical variants mentioned above, the hypertrophy or spasm of the piriformis muscles, local infectious events, spaceoccupying lesions (abscess, hematoma, tumors), Klippel Trenaunay syndrome, intramuscular injections and other traumatic events involving the gluteal region $(1,8)$. Many authors believe that local trauma followed by scarring is 
the most significant etiology of the syndrome, whilst anatomical variations can only predispose to this condition (8). Other structures, besides the piriformis muscle, may be involved in sciatic nerve entrapment within the gluteal space, such as fibrous bands containing blood vessels, gluteal muscles, hamstring muscles, the gemelli-obturator internus complex, vascular abnormalities. Therefore, the piriformis syndrome is only an example of the larger entity that is the "deep gluteal syndrome" (4).

The piriformis syndrome is a diagnosis of exclusion. In a systematic review, Hopayian et al. reported that there are four essential clinical features being most common for this syndrome: pain in the buttock aggravated while sitting, external tenderness near the greater sciatic notch, pain on any maneuver that increases piriformis muscle tension, and limitation of straight leg raising (9).

In his manuscript written in 1937, Freiberg described three criteria to identify piriformis-induced sciatica, as follows (5):

- Tenderness at the sciatic notch;

- Positive Lasègue sign;

- Improvement with nonsurgical treatment.

The first description of this syndrome as an entity, written by Robinson in 1947, enunciated six essential clinical aspects (10):

- A history of trauma to the sacroiliac and gluteal region;

- Pain in the region of the sacroiliac joint, greater sciatic notch and pyriformis muscle, extending down the leg and causing difficulty in walking;

- Acute exacerbation of the chronic pain brought on usually by stooping or lifting;

- The presence of a palpable sausage-shaped mass over the pyriformis muscle, which is tender to pressure during an acute exacerbation of pain. This clinical sign was considered almost pathognomonic;

- Positive Lasègue sign;

- Gluteal atrophy in cases where the condition evolves for a long period of time.

It has been argued that Freiberg's criteria are broad, lacking specificity, while Robinson's criteria are too specific, lacking sensitivity (11).

On physical examination of patients presenting with these symptoms, the initial evaluation should start with the lumbar spine, the most common origin of sciatica, and also take into account gait and posture. Examination of the hip, pelvis and sacroiliac joint should be performed (1). It is important to note that sensory and motor functions and deep tendon reflexes are typically normal (1). As mentioned above, evaluating the gluteal region often reveals tenderness to palpation of the piriformis muscle and in some cases even structural abnormalities. Internal palpation of the piriformis muscle to reveal tenderness, through vaginal or rectal exam, can be performed with greater sensitivity (12).
A series of clinical tests, such as Freiberg and FAIR maneuvers, can be performed to induce typical buttock pain, sciatica, weakness or paresthesia, through passive stretching of the piriformis muscle and subsequent compression of the sciatic nerve, in patients with piriform syndrome (5).

- In the Frieberg maneuver, the patient lies supine, while the examiner rotates the lower limb internally.

- In the FAIR test, the patient lies in supine position or on his side while the examiner places his hip in flexion, adduction and internal rotation.

Sciatica can also be induced in these patients by active contraction of the piriformis muscle (5).

- The active piriform test: the patient lies on his side and actively abducts and externally rotates the hip while the examiner resists these movements.

- The Beaty test: the patient lies on his side and holds their flexed hip in abduction against gravity.

- The Pace test: the patient is in a seated position and abducts his hip while the examiner resists this movement.

Lasègue maneuver is performed by passive flection of the hip with the knee in full extension, while the patient lies supine. The maneuver is not specific as it causes pain through the stretching of the sciatic nerve and not through compression by the piriformis muscle (5).

In 2013, Michel et al. (13) proposed a clinical scoring system for the diagnosis of piriformis syndrome that included 12 elements, each worth one point. A score that was equal or greater to 8 was considered "probable" in establishing the diagnosis. The criteria included were: unilateral or bilateral buttock pain with fluctuating periods without pain throughout the course of the day, sciatic pain of the same fluctuating character, the absence of lower back pain, no pain with axial spinal palpation (L2-S1), negative Lasègue sign, seated position that triggers buttock pain and/or sciatic pain, buttock pain next to the projection of the piriformis muscle that is reproduced by palpation, by active contraction or passive stretching of the piriformis muscle, sciatic pain reproduced by the same maneuvers, absence of perineal radiation. The sensitivity of this scoring system was $96.4 \%$ and its specificity $100 \%$. The positive predictive value was $100 \%$ and the negative predictive value was $86.9 \%$. While it is a noninvasive, easy to use and cost-effective way to evaluate patients in which a diagnosis of piriformis syndrome is suspected, it has not been validated in further studies (1). Imaging studies such as plain radiography, echography, computed tomography (CT-scan) and magnetic resonance imaging (MRI), as well as electroneuromyography can aid the clinician in evaluating these patients and establishing a diagnosis $(1,2,5)$.

- Pelvic radiography can identify calcifications in the piriformis muscle and, in rare cases, in the muscle tendon. It can also reveal bone abnormalities (14). 
- Soft tissue and musculoskeletal ultrasound is a noninvasive investigation that allows for the detection of muscular abnormalities and the local vasculature (8).

- CT-scan can be used in identifying the etiology by evaluating structural abnormalities such as spaceoccupying lesions (abscesses, hematomas, tumors) that lead to a compression of the sciatic nerve (5).

- MRI studies are usually preferred as they have greater sensitivity in evaluating soft tissue abnormalities, such as an enlarged piriformis muscle. It should be noted that enlargement of the piriformis muscle is not pathognomonic and is not always present in patients with piriformis syndrome (5). Moreover, piriformis muscle enlargement has been reported in about one fifth of asymptomatic patients. MRI neurography can enhance visualization of the peripheral nerves for a better evaluation (15).

- Electroneurography often shows normal results in these patients, but if maneuvers such as the active piriformis test, the Beatty test or the FAIR test are performed during the examination, a delay in the H-reflex can be observed and support the diagnosis. A delay greater than 1.86 msec during the FAIR maneuver is an electrophysiological criterion for the piriformis syndrome. Needle electromyography results are often normal, but denervation may be seen with longstanding nerve compression (16).

Treatment for piriformis syndrome can be conservative or surgical. Conservative treatment includes nonsteroidal anti-inflammatory drugs, muscle relaxants, agents used for neuropathic pain such as gabapentin, alongside physical therapy (1). Local steroid or anesthetic injections in the piriformis muscle are sometimes used. Misirlioglu et al. performed a double-blinded study in which they evaluated pain reduction when using local anesthetic with steroids versus local anesthetic alone. Improvements in pain was observed in both cases but no significant statistical difference was noted between the two groups (17). Botulinum toxin injections have recently been reported to alleviate pain in some patients by reducing the volume of the piriformis muscle $(16,18)$. Surgical intervention is to be used only in select cases that do not respond to a conservative approach, and consists of local decompression of the sciatic nerve (1). Current literature lacks sufficient data to justify a surgical treatment over the conservative approach, but there are multiple smaller studies that have reported a favorable outcome after surgery (1).

\section{Case presentation}

A 33-year-old Caucasian male, residing in a rural area, was admitted to our Neurology department with complaints of intense pain in the buttocks that spread in his left leg and was associated with weakness in the left lower limb and gait difficulty. The patient was an active smoker (1 pack of cigarettes per day for 20 years). He worked as a farmer and had no known allergies.

His symptoms had started 5 weeks prior to presentation when he was injured by a horse's hoof and suffered posterior joint dislocation of the left hip associated with ipsilateral acetabular fracture and cuboid fracture in his right foot. The dislocation was treated by an orthopedist with closed reduction followed by immobilization using a cast for 4 weeks. A few days after applying the cast the patient complained of intense pain (8-9 points on the numerical verbal scale) in his left buttock that spread in his left leg. He was given non opioid analgesics (Dexketoprofen $25 \mathrm{mg}$ per day) followed by weak opioid analgesics (Tramadol $75 \mathrm{mg}$ per day), which had little effect on the pain. After removing the cast, the pain persisted with the same intensity, which made physical therapy and rehabilitation impossible. During that period, he also developed an adjustment disorder with anxiety and suicidal ideation. He presented to our department one week after the cast was removed.

On physical examination the patient was conscious and aware, had normal body temperature, a BMI (Body Mass Index) of $19 \mathrm{~kg} / \mathrm{m}^{2}$. The blood pressure was $170 / 90$ $\mathrm{mmHg}$, and the heart rate 70 beats per minute.

The neurological examination revealed a lower motor neuron syndrome in the left lower limb characterized by paresis with a score of $2 / 5$ proximally and $0 / 5$ distally (on the MRC - Modified Research Council scale). The Achilles reflex in the left leg was also diminished and there was atrophy of the anterior compartment of the leg. There was intense tenderness of the greater sciatic notch alongside hypoesthesia, hyperalgesia and allodynia in the territory of the sciatic nerve. Lasègue sign was positive when the leg was raised at $30^{\circ}$ and the FAIR test and Freiberg's maneuver were both positive. The tests involving active contraction of the piriformis muscle could not be performed because of the motor deficit. According to the criteria proposed by Michel (13), our patient had a score of 9 points and, therefore, a "probable diagnosis" of piriformis syndrome. Regarding the patient's disability, he had a score of 4 on the Rankin scale (moderately severe disability) as he was unable to attend to own bodily needs without assistance and was unable to walk unassisted.

Complete bloodwork was done. No notable laboratory changes regarding the hematological profile, liver functions, kidney function, coagulation parameters were observed. No biological inflammatory syndrome was present during the time of admission.

Electroneurography and needle electromyography were performed and revealed a complete left sciatic nerve lesion.

MRI examination of the lumbar spine showed a left mediolateral disc protrusion at L1/L2 of $15 / 3 \mathrm{~mm}$ with 
consequent imprinting of the dural sac, narrowing of the lateral recess and radicular contact (Figure 1)

MRI examination of the pelvic region revealed edema of the left internal obturator muscle, marked edema and partial tearing of the external obturator muscle and a thin fluid collection along the upper border of the external obturator muscle, the head and neck of the femoral bone (Figure 2). There was also asymmetry regarding the piriformis muscle with contracture on the left side (Figure 3 ). MRI also showed diffuse edema of the femoral head and acetabulum on the left (Figures 2 and 3).
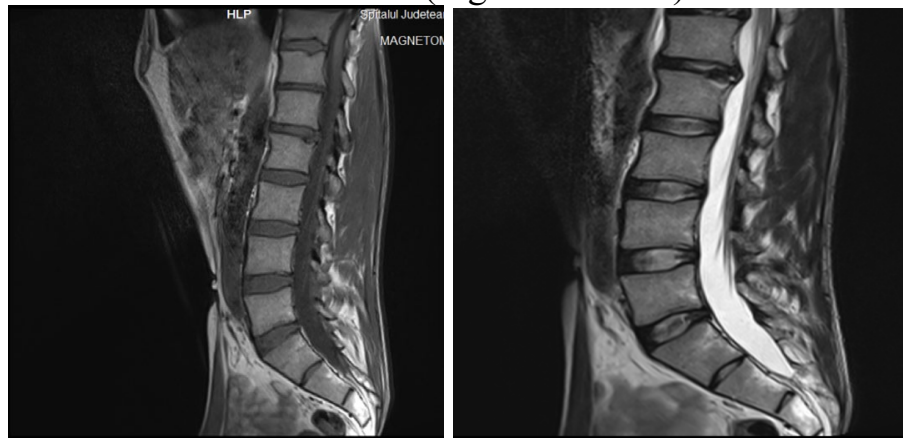

Figure 1: Sagittal cross-section MRI images of the lumbar spine, $\mathrm{T} 2$ and $\mathrm{T} 1$ weighted.

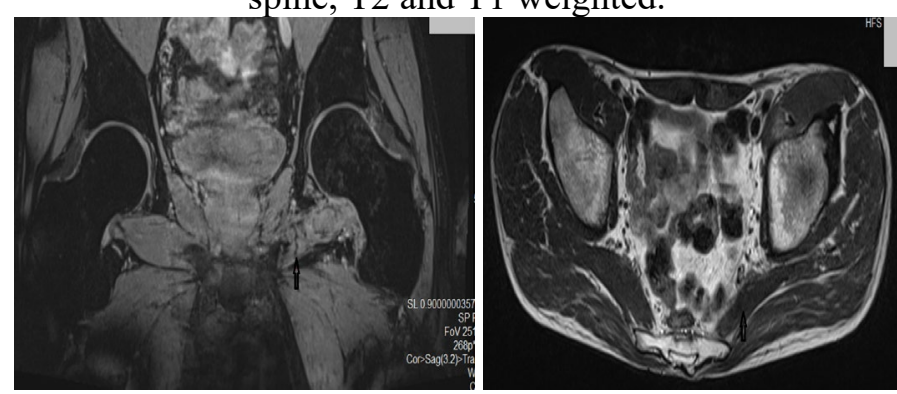

Figure 2: Coronal cross-section MRI images of the pelvic region, T2 weighted.

Figure 3. Axial cross-section MRI images of the pelvic region, $\mathrm{T} 1$ weighted.

As the patient continued to complain of intense pain that did not respond to NSAIDs and weak opioids (Tramadol), he was administered Gabapentin 300mg per day and a muscle relaxant/anxiolytic (Diazepam $20 \mathrm{mg}$ per day), with no success. A strong opioid was added afterwards (Fentanyl transdermal patch $50 \mu \mathrm{g} / \mathrm{h}$ which was later increased to $100 \mu \mathrm{g} / \mathrm{h}$ ). Regarding the adjustment disorder with anxiety and suicidal ideation, a psychiatric consultation was requested and he was given an antidepressant. Even after the administration of Fentanyl, the pain did not subside. Given the traumatic context and imaging aspects of the injury, intramuscular injection of anesthetics, steroids or botulinum toxin was ruled out and therefore a surgical approach to decompress the left sciatic nerve was considered and approved by a plastic surgeon.

The surgical intervention was performed under epidural anesthesia. During the intervention, the left sciatic nerve was found to be elongated and compressed by scar tissue, infra- and supragluteal, without any tearing in the nerve.
Surgical debridement of the scar tissue was performed (Figure 4). An epidural catheter with continuous administration of anesthetic was placed for 48 hours after the surgery.

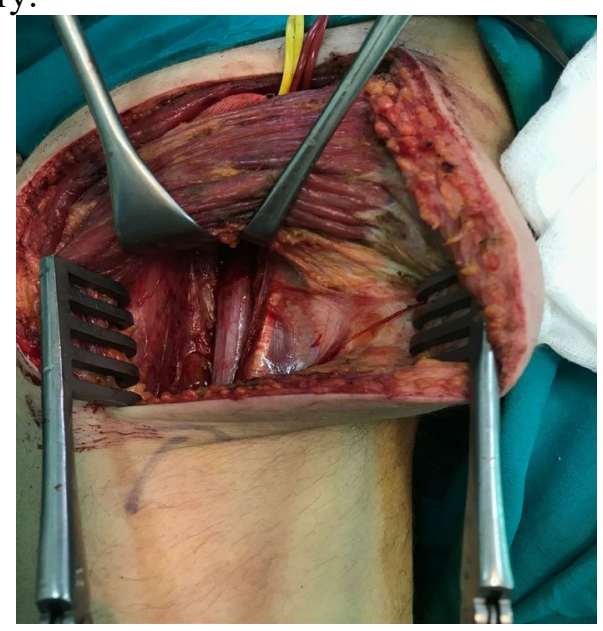

Figure 4: Intraoperative aspect of the left sciatic nerve after the debridement of the scar tissue.

After surgery, the pain started to subside and could be managed with Tramadol (75 mg per day) and Gabapentin (600 mg per day). The muscle strength improved and the patient regained partial movement of the foot and toes. He was able to stand up without support at 3 days after intervention, but walking remained impossible (about 2 or 3 steps with unilateral support).

One month after surgery, the patient began a rehabilitation program that included physical exercise, therapeutic massage and complementary pain treatment (TENS, exponential currents, galvanic currents, pulsed electromagnetic energy). There was a favorable evolution regarding the pain (3-4 points on the numerical verbal scale), but the motor deficit persisted. An ankle-foot orthosis fixed with a 90-degree bend at the ankle was used. The patient was able to walk using an assistive cane for the first 4 weeks, and without any support afterwards. The Rankin score improved, with a score of 2 points (slight disability).

\section{Discussion}

Pain was the main cause for disability in the case we presented and could only be managed using a multidisciplinary approach, namely pharmacological agents, surgery and complementary pain treatment.

Pain that is caused by central or peripheral nervous system dysfunction, which persists in the absence of noxious stimuli, meets the criteria for neuropathic pain (19). The International Association for the Study of Pain (IASP) defines neuropathic pain as pain caused by a lesion or disease of the somatosensory nervous system (20). This definition was used to reflect the pathophysiological mechanism and also to enunciate the fact that neuropathic pain is not a disease, but a consequence of a larger group of diseases, each with various signs and symptoms (21). 
A system was proposed to determine the level of certainty that qualifies pain to be neuropathic, as follows (22):

- Possible - if the patient's history suggests the presence of a neurological lesion or disease and the pain could be related to it (for example, using validated screening tools) and the pain distribution is neuroanatomically plausible;

- Probable - requires supporting evidence obtained by a clinical examination of sensory signs (for example, bedside testing and quantitative sensory testing);

- Definite - requires that an objective diagnostic test confirms the lesion or disease of the somatosensory nervous system (for example, neurophysiological tests and skin biopsy).

In our case, electroneurographic testing as well as intraoperative findings confirmed the lesion of the sciatic nerve. The pain was also distributed in the territory of innervation of the sciatic nerve.

There are multiple pathophysiological mechanisms that contribute to neuropathic pain such as: peripheral and central sensitization, central reorganization of the somatosensory cortex after peripheral nerve lesions, ectopic discharges originated from injured sites, loss of intrinsic inhibitory control (23).

Following a peripheral nerve injury, a series of ectopic discharges are generated through the altered function of the sodium channels. Peripheral neuropathy alters the electrical properties of sensory nerves, causing imbalances between central excitatory and inhibitory signaling, which lead to the impairment of inhibitory interneurons and descending control systems. In turn, transmission of sensory signals and disinhibition or facilitation mechanisms are altered at the level of the spinal cord dorsal horn neurons (22). Moore et al. (24) have found a loss of postsynaptic GABA-A receptormediated inhibition in the superficial dorsal horn in two different partial peripheral nerve injury models. Because similar changes did not occur after a complete section of the sciatic nerve, it appears that some intact input is required for the loss of inhibition to occur.

In neuropathic pain, plasticity of the neurons in the spinal cord plays a key role in abnormal signaling that leads to pain in the absence of noxious stimuli. Glial cells, such as astrocytes contribute to this plasticity through the following factors (23):

- An excellent control of synaptic concentrations of neurotransmitters and gliotransmitters such as D-serine, ATP and glutamate;

- Synthesis of neurotrophic factors that influence the synaptic micro-climate, promote axonal growth;

- Intercellular communication with neurons through specific receptor-ligand interactions.

Regarding the spinal glutaminergic system, it has been shown that glutamate signaling is altered in patients with peripheral nerve injury. Astrocytes have specific GTs (Glutamate Transporters) that regulate the uptake of endogenous glutamate. In rats it has been shown that after chronic constrictive nerve injury, the expression and uptake activity of spinal GTs changed and contributed to neuropathic pain behaviors (25).

Consequently, the brain receives altered and abnormal sensory messages. Altered projections to the thalamus and cortex and parallel pathways to the limbic regions account for high pain ratings and anxiety, depression and sleep problems, which are relayed as painful messages that dominate the limbic function. Areas such as the cingulate cortex and amygdala have been implicated in the ongoing pain state and comorbidities associated with neuropathic pain (22). Most patients with neuropathic pain express one of two pro-nociceptive pain modulation profiles, by which pain messages are augmented in the CNS. These profiles could explain why the patients respond differently to medication (22):

- Patients with a facilitating pronociceptive profile, in which the perception of pain is facilitated through sensitization of ascending pain pathways. These patients could be better treated with a drug that reduces facilitation (such as gabapentinoids);

- Patients with an inhibitory pro-nociceptive profile, in which the perception of pain is disinhibited due to decreased descending endogenic inhibition. These patients could be better treated with a drug that enhances inhibition (such as serotonin-noradrenaline reuptakeinhibitors).

Apart from pharmacological treatment, various complementary pain treatment methods can be used, such as Transcutaneous electrical nerve stimulation - TENS, therapeutic massage and psychological support. TENS is a non-invasive peripheral stimulation technique used to relieve pain. During TENS pulsed electrical currents are delivered across the intact surface of the skin to activate underlying nerves (26). Even though TENS is frequently used for pain management, there is not much evidence to support its effectiveness (27). In our case, TENS was used alongside pharmacological treatment and after surgery, and seemed to have a positive effect on relieving pain but, as it was only used in combination with other methods of treatment, we cannot quantify its individual effect on reducing pain.

\section{Conclusions}

In our case, the sciatic nerve lesion was not only caused by the injured piriformis muscle but also by adjacent scarring and surrounding damaged muscles, and therefore a diagnosis of deep gluteal syndrome would have seemed more appropriate. However, we considered that the diagnosis of piriformis syndrome was better suited as the name was initially conceived to specifically describe a posttraumatic event in which the main mechanism of injury implies the compression of the nerve by the piriformis muscle, which was true in our case. 
The rehabilitation program had an important role in relieving pain for our patient, increasing muscle strength and improving the general quality of life, through physical therapy, orthopedic devices, pain medication and complementary pain therapy.

Informed consent - An informed consent was obtained from the patient participating in the study.

\section{Declaration of conflict of interests}

The authors declare that there was no conflict of interest regarding the publication of this paper.

\section{Author contributions.}

1. Vitalie Văcăraș: head of department, consultant neurologist in charge of the patient. Given approval of the final version.

2. Sorina Frunze: resident doctor, conception and design of the case report.

3. Adrian Mihai Cordoș: resident doctor, revising the case report and drafting the discussion section

4. Tatiana Perciuleac: resident doctor, revising the case report and comparing it to the current literature

5. Dafin Fior Mureșanu: coordinator of the team.

\section{Acknowledgements.}

Many thanks to Ioana Robu, Iuliu Hatieganu University of Medicine and Farmacy of Cluj, Romania, for the help in editing this manuscript

\section{References:}

1. Cass SP. Piriformis syndrome: a cause of nondiscogenic sciatica. Curr Sports Med Rep. 2015 Jan;14(1):41-4.

2. Hopayian K, Song F, Riera R, Sambandan S. The clinical features of the piriformis syndrome: a systematic review. Eur Spine J. 2010 Dec;19(12):2095-109.

3. Robinson DR. Pyriformis syndrome in relation to sciatic pain. Am J Surg. 1947 Mar;73(3):355-8.

4. Martin HD, Reddy M, Gomez-Hoyos J. Deep gluteal syndrome. Journal of Hip Preservation Surgery. 2015 Jul 1;2(2):99-107.

5. Probst D, Stout A, Hunt D. Piriformis syndrome: a narrative review of the anatomy, diagnosis, and treatment. PM R. 2019;11 Suppl 1:S54-63.

6. Beaton LA, Anson BJ. The sciatic nerve and the piriformis muscle: their interrelation a possible cause of coccygodynia. $\mathrm{J}$ Bone Joint Surg. 1938 Jul;20(3):686-688.

7. Windisch G, Braun EM, Anderhuber F. Piriformis muscle: clinical anatomy and consideration of the piriformis syndrome. Surg Radiol Anat. 2007 Feb;29(1):37-45.

8. Jankovic D, Peng P, van Zundert A. Brief review: piriformis syndrome: etiology, diagnosis, and management. Can J Anaesth. 2013 Oct;60(10):1003-12.

9. Hopayian K, Danielyan A. Four symptoms define the piriformis syndrome: an updated systematic review of its clinical features. Eur J Orthop Surg Traumatol. 2018 Feb;28(2):155-64.

10. Freiberg AH. Sciatic pain and its relief by operations on muscle and fascia. Arch Surg. 1937 Feb 1;34(2):337.

11. Fishman LM, Dombi GW, Michaelsen C, Ringel S, Rozbruch J, Rosner B, et al. Piriformis syndrome: diagnosis, treatment, and outcome--a 10-year study. Arch Phys Med Rehabil. 2002 Mar;83(3):295-301.

12. Durrani Z, Winnie AP. Piriformis muscle syndrome: an underdiagnosed cause of sciatica. J Pain Symptom Manage. 1991 Aug;6(6):374-9.

13. Michel F, Decavel P, Toussirot E, Tatu L, Aleton E, Monnier $\mathrm{G}$, et al. Piriformis muscle syndrome: diagnostic criteria and treatment of a monocentric series of 250 patients. Ann Phys Rehabil Med. 2013 Jul;56(5):371-83.

14. Stark P, Hildebrandt-Stark HE. Calcific tendinitis of the piriform muscle. Rofo. 1983 Jan;138(1):111-2.

15. Lewis AM, Layzer R, Engstrom JW, Barbaro NM, Chin CT. Magnetic resonance neurography in extraspinal sciatica. Arch Neurol. 2006 Oct;63(10):1469-72.

16. Fishman LM, Wilkins AN, Rosner B. Electrophysiologically identified piriformis syndrome is successfully treated with incobotulinum toxin a and physical therapy. Muscle Nerve. 2017 Apr;56(2):258-63.

17. Misirlioglu TO, Akgun K, Palamar D, Erden MG, Erbilir T. Piriformis syndrome: comparison of the effectiveness of local anesthetic and corticosteroid injections: a double-blinded, randomized controlled study. Pain Physician. 2015 Apr;18(2):163-71.

18. Al-Al-Shaikh M, Michel F, Parratte B, Kastler B, Vidal C, Aubry S. An MRI evaluation of changes in piriformis muscle morphology induced by botulinum toxin injections in the treatment of piriformis syndrome. Diagn Interv Imaging. 2015 Jan;96(1):37-43.

19. Treede R-D, Jensen TS, Campbell JN, Cruccu G, Dostrovsky JO, Griffin JW, et al. Neuropathic pain: redefinition and a grading system for clinical and research purposes. Neurology. 2008 Apr 29;70(18):1630-5.

20. Iasp terminology - iasp [Internet]. [cited 2020 Nov 25]. Available from: https://www.iasppain.org/Education/Content.aspx?ItemNumber $=1698$

21. Jensen TS, Baron R, Haanpää M, Kalso E, Loeser JD, Rice ASC, et al. A new definition of neuropathic pain. Pain. 2011 Oct;152(10):2204-5.

22. Colloca L, Ludman T, Bouhassira D, Baron R, Dickenson AH, Yarnitsky D, et al. Neuropathic pain. Nat Rev Dis Primers. 2017 Feb 16;3:17002.

23. Motoc D, Turtoi NC, Riga D, Riga S. Neuropathic pain physiological and physiopathological features. Therapeutic trends. Revista Medicală Română. 2011;58(1):21-27.

24. Moore KA, Kohno T, Karchewski LA, Scholz J, Baba H, Woolf CJ. Partial peripheral nerve injury promotes a selective loss of GABAergic inhibition in the superficial dorsal horn of the spinal cord. J Neurosci. 2002 Aug 1;22(15):6724-31.

25. Sung B, Lim G, Mao J. Altered expression and uptake activity of spinal glutamate transporters after nerve injury contribute to the pathogenesis of neuropathic pain in rats. J Neurosci. 2003 Apr 1;23(7):2899-910.

26. Johnson M. Transcutaneous electrical nerve stimulation: mechanisms, clinical application and evidence. Rev Pain. 2007 Aug;1(1):7-11.

27. Gibson W, Wand BM, O'Connell NE. Transcutaneous electrical nerve stimulation (Tens) for neuropathic pain in adults. Cochrane Database Syst Rev. 2017 Sep 14;9:CD011976 